\title{
WHY WE FEAR THE WRONG THINGS?
}

\section{WARUM WIR UNS VOR DEN FALSCHEN DINGEN FÜRCHTEN?}

\author{
Alwin Schönberger \\ Vienna, Austria
}

\section{SUMMARY}

Deadly shark attacks, cancer from meat, toxic beer: Our health seems to be threatened seriousely all the time. But do we fear the real dangers? And how can we identify the big risks? How to deal with numbers and statistics.

Key words: statistics - absolute risk - relative risk - cancer and evironmental dangers - correlation - causation - risk communication

\section{ZUSAMMENFASSUNG}

Tödliche Haiattacken, Krebs durch Fleisch, giftiges Bier: Ständig scheint unsere Gesundheit ernsthaft bedroht zu sein. Doch fürchten wir uns vor den wirklichen Gefahren? Und wie erkennt man ein großes Risiko? Über den Umgang mit Zahlen und Statistiken.

Schlüsselwörter: Statistik - absolutes Risiko - relatives Risiko - Krebs- und Umweltgefahren - Korrelation Kausalität-Risikokommunikation
Stellen Sie sich vor, Sie fragen zwei Personen nach dem Weg. Die erste sagt: Gehen Sie ungefähr einen Kilometer geradeaus, dann links, ein kleines Stück gerade und dann ziemlich bald rechts. Die zweite Person sagt: Sie gehen 1200 Meter geradeaus, scharf rechts, 250 Meter gerade, dann nochmals rechts, und nach 20 Metern sind Sie da. Welcher Auskunft glauben Sie? Mit hoher Wahrscheinlichkeit vertrauen Sie eher der zweiten Person, weil sie viel exaktere Angaben liefert. Der Statistikprofessor Walter Krämer aus Dortmund nennt dies die Illusion der Präzision: Je genauer die Zahlen sind, die uns vermittelt werden, desto eher nehmen wir an, dass sie korrekt sind - was aber keineswegs stimmen muss.

Doch Zahlen, besonders wenn sie säuberlich bis weit in den Nachkommabereich aufgelistet sind, erwecken den Anschein von Vertrauenswürdigkeit. Zahlen kommen uns außerdem objektiv und unbestechlich vor. Grundsätzlich stimmt das natürlich auch. Ziffern und Statistiken ergreifen nicht Partei, verfolgen keine Absichten, sondern bilden einfach Gegebenheiten oder Entwicklungen ab. Dennoch können sie uns manchmal gewaltig in die Irre führen - nämlich dann, wenn wir uns mit der Interpretation plagen. Mit „uns“ sind fast alle Menschen gemeint, Mathematiker inbegriffen. Die meisten von uns tun sich chronisch schwer, mit nackten, nüchternen Zahlen angemessen umzugehen. Der Mensch scheint, das bestätigen zahlreiche Experten, schlichtweg nicht dafür gemacht, in Zahlen zu denken. Die Bedeutung von Ziffern erschließt sich uns nicht intuitiv, wir müssen uns deren Bedeutung von Fall zu Fall mühselig erarbeiten. Das können wir kaum ändern - aber wir sollten uns dieser Schwäche bewusst sein.

Das Problem stellt sich schon bei kleinen, harmlosen Rätseln des Alltags. Was zum Beispiel heißt 30 Prozent
Regenwahrscheinlichkeit? Muss man davon ausgehen, dass es ungefähr ein Drittel der Zeit in diesem Tag regnen wird? Oder auf einem Drittel der Fläche, für die die Prognose gilt? Oder bedeutet es vielmehr, dass es an 30 Prozent aller Tage regnet, an denen die Wetterlage so beschaffen ist wie am jenem, für den die Vorhersage erstellt wurde?

Vermutlich haben Sie die richtige Antwort allein aufgrund der sperrigen Formulierung der Variante drei erraten. Wenn Sie trotzdem unsicher waren: Trösten Sie sich, Sie sind in guter Gesellschaft. Dabei sind wir ständig mit Zahlen und Statistiken konfrontiert. Sobald wir die Zeitung aufschlagen, springen uns Zahlen entgegen, mit denen versucht wird, Sachverhalte zu untermauern, Tendenzen abzuleiten, die Wirtschaftslage darzustellen und Behauptungen generell einen soliden und seriösen Anschein zu verleihen.

Sehr häufig begegnen uns Statistiken in Zusammenhang mit Risiken des Alltags: Gifte, Krankheiten, Umweltgefahren, Unfälle, Toxizität von Nahrung, Häufigkeit von Anschlägen - all das wird gerne in Zahlen ausgedrückt. Fürchten wir uns aber vor den wirklichen Gefahren? Eher nein, und das hat zum einen psychologische Gründe. Wir haben Angst vor sogenannten Schockrisiken, zum Beispiel vor einer Terrorattacke oder einem angeblichen Killerasteroiden. Das sind Ereignisse, bei denen zur selben Zeit an einem Ort viele Menschen gemeinsam ums Leben kommen. Experten vermuten dahinter dafür evolutionäre Ursachen: Der Mensch fürchtet sich vor solchen Großereignissen, weil sie einen erheblichen Teil seiner Sippe auf einen Schlag auslöschen können.

Wir fürchten uns dagegen weniger vor dem Straßenverkehr, obwohl natürlich die Wahrscheinlichkeit viel höher ist, bei einem Verkehrsunfall zu verunglücken als durch einen Asteroideneinschlag. Wir ängstigen uns 
außerdem vor Dingen, die von außen kommen, fremdverschuldet sind und sich unserem Einfluss entziehen, zum Beispiel vor Gehirntumoren durch Mobilfunkstrahlen. Dabei wird in Österreich bei gerade mal rund 600 Menschen pro Jahr ein solcher Tumor diagnostiziert, sämtliche Auslöser inbegriffen. Demgegenüber sterben mehr als 30.000 Österreicher jährlich an HerzKreislauf-Leiden, denen man durch eine Änderung des Lebensstils begegnen könnte. Diese Ziffern versetzen aber kaum jemanden in Panik.

Die verzerrte Einschätzung von Risiken ist aber nicht nur ein psychologisches Problem, sondern auch eines des Zahlenverständnisses. Mit eindrucksvollen Zahlen und nach oben schnellenden Kurven wird regelmäßig versucht, das Ausmaß einer Bedrohung anschaulich darzustellen - nicht nur von Zeitungen, sondern gar nicht so selten von Wissenschaftlern in anerkannten Fachjournalen. Es ist aber für den Laien trotz präziser Ziffern oft gar nicht leicht zu erkennen, ob nun ein Risiko groß oder klein ist. Anders ausgedrückt: Zahlen können vollkommen korrekt sein und trotzdem ein falsches Bild vermitteln. Zahlen können Ängste schüren und großflächige Furcht hervorrufen, auch wenn bei näherer Betrachtung nicht unbedingt ein Anlass dafür besteht. Wir müssen sie also richtig lesen lernen, um aus ihnen schlau zu werden.

Der klassische Fall dafür, wie man mit richtigen Zahlen einen falschen Eindruck erwecken kann, ist die (gedankenlose oder aber gezielte) Verwendung relativer statt absoluter Risiken. Weist man Gefahren in Prozent statt in absoluten Zahlen aus, kann das leicht zu Trugschlüssen und einer grob verzerrten Risikoeinschätzung führen.

Ein Beispiel: Vor einigen Jahren hieß es, die Zahl der tödlichen Haiangriffe sei um 43 Prozent gestiegen. Das klang bedrohlich, und man mochte sich bange fragen: Was ist bloß in die Haie gefahren? Gar nichts. Es gibt bloß so extrem wenige Haiangriffe, dass sich ein paar Fälle mehr, also ein kleiner Ausreißer nach oben, in einer gewaltigen prozentualen Steigerung niederschlägt (Abbildung 1).

Prozentangaben können also, nicht nur in diesem Fall, ziemlich ireführend sein. Die absoluten Zahlen zeigen hingegen, dass die Gefahr einer tödlichen Haiattacke völlig vernachlässigbar ist. Dennoch: Beide Angaben sind korrekt, aber die Prozentzahl erweckt einen gänzlich falschen Eindruck.

Misstrauen Sie Prozentangaben grundsätzlich. Fragen Sie immer nach den absoluten Zahlen. Worauf bezieht sich eine prozentuale Steigerung? Um wie viel Prozent wovon geht es überhaupt, was ist die Basis der Berechnung? Wichtig ist immer das absolute Risiko, nicht das relative. Im konkreten Fall beträgt das relative Risiko 43 Prozent, das absolute aber nur drei. Basis sind die sieben Fälle des Jahres 2012, und diese Basis muss man kennen, um ein Risiko überhaupt sinnvoll beurteilen zu können. Nur drei Fälle mehr bedeuten in diesem Fall trotzdem ein Plus von 43 Prozent.
Haiangriffe weltweit

\begin{tabular}{|l|l|l|}
\hline Jahr & Gesamt & $\begin{array}{l}\text { Davon } \\
\text { tödliche } \\
\text { Angriffe }\end{array}$ \\
\hline 2012 & 83 & 7 \\
\hline 2013 & 75 & $\left(\begin{array}{c}7 \\
10\end{array}\right.$ \\
\hline 2014 & 72 & 3 \\
\hline
\end{tabular}

\section{$3 / 7=43 \%$}

Abbildung 1. Haiangriffe

Bedauerlicherweise benutzen sogar Wissenschaftler manchmal immer noch die verwirrenden relativen Risikoberechnungen, obwohl sie es besser wissen müssten. Sie erzeugen damit, aus welchen Motiven immer, einen falschen Eindruck vom Ausmaß einer Gefahr. Besonders heftig debattiert wurde vor einiger Zeit folgendes Risiko, das durch alle Medien ging und ziemliche Unruhe ausgelöst hat: Wer gerne Wurst oder rotes Fleisch isst, hat laut Weltgesundheitsorganisation ein um 18 Prozent erhöhtes Darmkrebsrisiko. Das klingt gefährlich, aber was steckt dahinter? Erkrankt fast ein Fünftel aller Fleischesser an Darmkrebs? Stirbt gar beinahe ein Fünftel daran?

Um uns ein Bild zu machen, sollten wir zwei Dinge tun: Erstens lassen wir die Prozentzahlen beiseite und fragen - was man in solchen Fällen immer tun sollte nach ,echten“ Menschen, also nach der Grundgesamtheit, somit neuerlich nach jener Basis, auf der die Berechnung fußt (genau die wird in solchen Debatten allerdings häufig nicht erwähnt oder geflissentlich ignoriert). Stellen wir uns eine Gruppe von 100 Personen vor. Um zu wissen, ob die behaupteten 18 Prozent viel oder wenig sind, müssen wir nun zuerst fragen: Wie viele von diesen Personen bekommen im Schnitt die Diagnose Darmkrebs, einfach so, aus welchen Gründen auch immer?

Das sind, so zeigen Gesundheitsdaten, etwa drei von 100 Personen - also zum Glück nicht sehr viele (wobei wir von Diagnosen reden, nicht von tatsächlichen Erkrankungen; es sind also auch Personen dabei, bei denen sich der Anfangsverdacht nicht bestätigte). Nun müssen wir in Erfahrung bringen: Um wie viele mehr bekommen eine Darmkrebsdiagnose, weil sie Fleisch essen? Diese Frage ist entscheidend, denn es erhalten ja, wenn man genau liest, nicht 18 Prozent der Menschen solch einen Befund, sondern um 18 Prozent mehr Personen, als sonst betroffen wären. Daher ist die Frage: Um wie viel mehr sind 18 Prozent von drei Personen? Das Ergebnis lautet: 0,54. Die beiden Angaben 18 Prozent beziehungsweise 0,54 bedeuten exakt dasselbe, klingen aber schon sehr verschieden. Das heißt nicht, dass man bedenkenlos Fleisch essen sollte. Aber 0,54 mehr erweckt doch einen ganz anderen Eindruck als 18 Prozent mehr (Abbildung 2). 


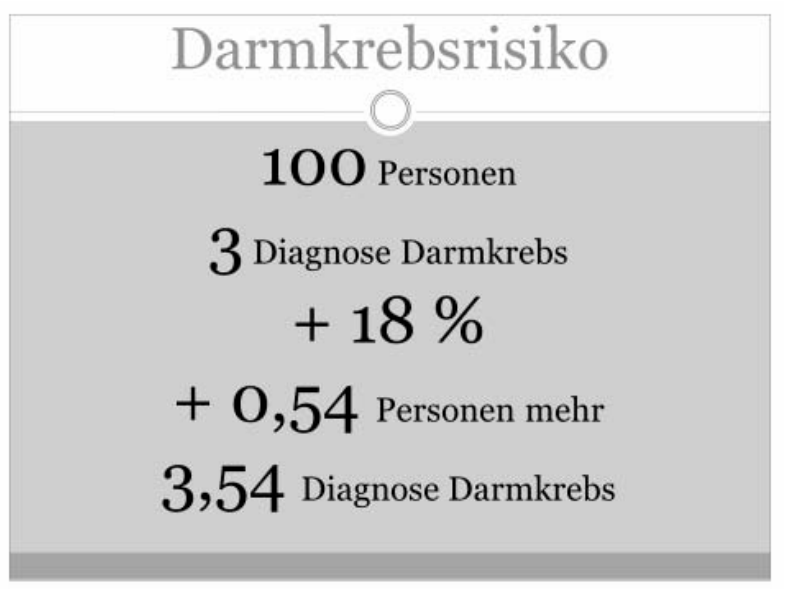

Abbildung 2. Krebs durch Fleisch

Diesem Problem begegnen wir eigentlich ständig: der oft krassen Diskrepanz zwischen absoluten und relativen Risiken. Wir sollten daher immer fragen: Wie viele Personen sind tatsächlich wovon betroffen? Dann können wir viel leichter beurteilen, welches Ausmaß eine bestimmte Gefahr erreicht. Das ist auch deshalb wichtig, weil die Kommunikation relativer Risiken durchaus bedenkliche Folgen haben kann, wenn überzogene Ängste geschürt werden. Als vor längerer Zeit die sogenannte dritte Generation der Antibabypille auf den Markt kam, ging eine Schockmeldung durch die Länder: Die Einnahme erhöhe das Risiko für Thrombosen um 100 Prozent. Eine Verdoppelung der Gesundheitsgefahr klingt natürlich wirklich alarmierend. Was aber bedeuteten doppelt so viele Thrombosen in absoluten Zahlen? Wenden wir dieselbe Methode an wie zuvor. Fragen wir, wie viele Frauen normalerweise eine Thrombose bekommen - und wie viele mehr eine solche erleiden, weil sie die neue Pille einnehmen.

Der Berliner Bildungs- und Risikoexperte Gerd Gigerenzer hat diese Rechnung durchgeführt und damit das tatsächliche Risiko ermittelt, das von der Pille ausgeht. Im Durchschnitt erleidet (aus unterschiedlichsten Gründen) eine unter 7000 Frauen eine Thrombose. Wenn sich diese Zahl durch die Einnahme verdoppelt - also eine Steigerung von 100 Prozent eintritt kommen wir auf zwei Frauen, also eine immer noch sehr kleine Zahl. Und neuerlich gilt: Zwei statt eine von 7000 klingt doch ganz anders als plus 100 Prozent, obwohl beide Ziffern korrekt sind. Wenn der Absolutwert klein ist, bleibt auch die Steigerung gering, fast einerlei, wie groß der prozentuale Anstieg ist (Abbildung 3).

Das Tragische an der konkreten Geschichte: Infolge der Meldungen über ein um 100 Prozent erhöhtes Thromboserisiko setzte eine wahre Pillenpanik ein. Es kam zu Abtreibungen, ungewollten Schwangerschaften und zahlreichen medizinischen Komplikationen, deren Ausmaß das Thromboserisiko weit überstieg.

Doch es gibt auch andere Methoden, um Risiken größer erscheinen zu lassen als sie tatsächlich sind - und Menschen über deren Dimension in die Irre zu führen. Ein Rezept dafür lautet: Man verwende Maßeinheiten, unter denen sich kein Mensch etwas vorstellen kann.

\section{Thrombose durch Antibabypille}

1 von 7000 Frauen

$$
\begin{gathered}
+100 \%=+1 \text { Frau } \\
2 \text { von } 7000 \text { Frauen }
\end{gathered}
$$

Abbildung 3. Thrombosen

Und man unterlasse eine Umrechnung in Messgrößen, die unserem Alltagsverstand ein Gefühl für das Ausmaß der behaupteten Gefahr vermitteln. Wieder stellvertretend dafür ein Beispiel, das heftig diskutiert wurde und Ängste erzeugt hat: Unser Bier ist mit Glyphosat verseucht.

Tatsächlich wurde bei Tests das Unkrautvernichtungsmittel gefunden, und zwar vier bis sieben Mikrogramm Glyphosat pro Liter Bier. Nun kann man zu Recht sagen, Gift hat in Lebensmitteln grundsätzlich nichts verloren. Trotzdem ist die Frage interessant, ob nun etwa vier Mikrogramm Glyphosat viel oder wenig sind und ob dadurch gesundheitliche Gefahren drohen. Vergleichen wir zu diesem Zweck den Glyphosatgehalt mit einem anderen Gift im Bier, das wir gerne und freiwillig konsumieren: Alkohol. Etwa fünf Prozent enthält Bier im Schnitt. Um einen direkten Vergleich mit der Anreicherung mit Glyphosat anstellen $\mathrm{zu}$ können, ist es nützlich $\mathrm{zu}$ wissen, wieviel Mikrogramm das sind. Es sind, so unglaublich das erscheinen mag, 40 Millionen Mikrogramm. Ein Liter Bier enthält also ein paar Mikrogramm Glyphosat, aber 40 Millionen Mikrogramm Alkohol! (Abbildung 4).

Jetzt lässt sich ungefähr abschätzen, wie groß die Wahrscheinlichkeit ist, sich beim Biertrinken mit Glyphosat zu vergiften: Bevor das geschieht, wäre der Biertrinker längst einer Alkoholvergiftung erlegen. Wenn Sie sich also vor fünf Prozent Alkohol nicht fürchten, gibt es wenig Grund, Angst vor dem Biertrinken zu haben, außer Sie trinken ein paar hundert Liter am Tag. Dann aber ist Glyphosat Ihr kleinstes Problem. Die Messergebnisse sind aber aus einem anderen Grund bemerkenswert: Sie stellen einen Triumph moderner, hochsensibler Analytik dar früher hätte man derart geringe Mengen überhaupt nicht nachweisen können. Aus diesem Grund sind Warnungen vor kontaminierten Lebensmitteln heute oft irreführend: Dass Toxine darin entdeckt werden, liegt nicht daran, dass die Lebensmittel häufiger mit Schadstoffen belastet sind als früher, sondern vor allem daran, dass man sie mit den heutigen Messmethoden aufspüren kann - früher wäre das gar nicht möglich gewesen. 


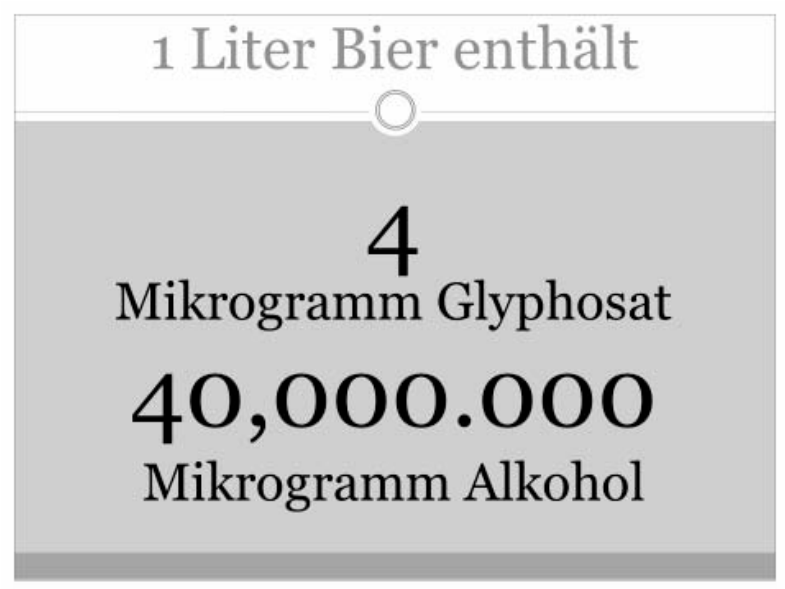

Abbildung 4. Glyphosat

Noch ein weiteres Beispiel zu diesem Themenkomplex. Vor einiger Zeit gab es helle Aufregung über Emissionen von Dieselruß. 170 Menschen in Österreich würden jährlich an den Abgasen sterben. Natürlich ist jedes Todesopfer beklagenswert, und man sollte nie Tote gegeneinander aufrechnen, aber um die Dimension eines Problems zu erfassen, ist es mitunter schon $\mathrm{zu}-$ lässig, solch eine Zahl mit anderen Gefahren zu vergleichen. Ist der Dieselruß nun eine große Gefahr oder nicht?

Im Jahr 2016 gab es beispielsweise 427 Verkehrstote. Selbst beim Bergsteigen droht erhebliche Lebensgefahr: Mehr als 250 tödliche Bergunfälle ereigneten sich im selben Jahr - eigentlich verblüffend viele. Wenn wir nun die Opfer durch Dieselruß näher betrachten, ist eine Detailrechnung nötig: Die Autoren die Studie wollten nicht zuletzt darauf hinweisen, dass die technischen Manipulationen der Abgaswerte an erhöhtem Schadstoffausstoß und damit an zusätzlichen Totesopfern Schuld trugen. Eine zentrale Frage war daher: Wie viele Todesfälle wären vermeidbar gewesen, hätten die kriminellen Tricksereien der Konzerne nicht stattgefunden? Die Studie ging von 50 Prozent vermeidbaren Todesfällen aus. Will man wissen, wieviele Menschen normalerweise durch Dieselabgase versterben, wie groß somit das Gesundheitsrisiko durch Dieselruß üblicherweise ist, muss man den Anstieg durch die Manipulationen wegrechnen. Dann kommt man auf die Zahl der Menschen, die allein deshalb versterben, weil Österreichs Straßen von Dieselautos befahren werden. Das Ergebnis dieser Rechnung lautet: 80 bis 90 Personen pro Jahr erliegen Atemwegsleiden, weil Autos mit Diesel betrieben werden. Selbstverständlich ist das bedauerlich, aber gemessen an der Verkehrsdichte und anderen Gefahren ist dieses Risiko doch vergleichsweise gering.

Hier sind Ursache und Wirkung wenigstens miteinander verknüpft. Es handelt sich um sogenannte kausale Zusammenhänge. Das ist aber keineswegs immer der Fall. Eine der größten und häufigsten Fallen bei Statistiken ist es, zwei Dinge zu verbinden, die gar nichts miteinander zu tun haben, außer dass zwei Kurven parallel zueinander verlaufen. Ständig begegnen wir in den Zeitungen vermeintlichen Ursachen von Gefahren, die sich nur durch blanke Korrelation ergeben. Stellvertretend ein Beispiel für diese beständige Flut von Meldungen, die meist bestimmte Lebens- oder Verhaltensweisen zu erwartenden Risiken gegenüberstellen: Akademiker bekommen eher Hirnkrebs, besonders dann, wenn sie verheiratet sind. Ist es gesundheitsschädlich, zu studieren und zu heiraten? Ziemlich unwahrscheinlich, hier treten vermutlich bloß drei Faktoren aufeinander - Ausbildung, Ehe, Krebs -, die miteinander ursächlich aber nichts zu tun haben. Man sollte stets sehr skeptisch sein, wenn einem solche Schlagzeilen begegnen, was freilich nahezu täglich der Fall ist.

Manchmal sind die Erklärungen für solch scheinbar frappierenden Zusammenhänge recht banal. Ein Beispiel dafür: Je weniger Haare man auf dem Kopf hat, desto höher ist das Einkommen. Wird man aber tatsächlich reicher, weil die Haare ausfallen? Das würde wohl niemand ernsthaft annehmen. Des Rätsels simple Lösung: Mit zunehmendem Lebensalter verlieren nun mal viele Menschen ihre Haare. Zugleich erwerben sie mehr Wohlstand, weil sie über die Jahre in höhere berufliche Positionen aufrücken. Es handelt sich eben um eine bloße Korrelationen: Zwei Entwicklungen verlaufen gleichzeitig und parallel zueinander, obwohl sie ursächlich miteinander nichts zu tun haben.

\section{Number of people who drowned by falling into a pool correlates with}

\section{Films Nicolas Cage appeared in}

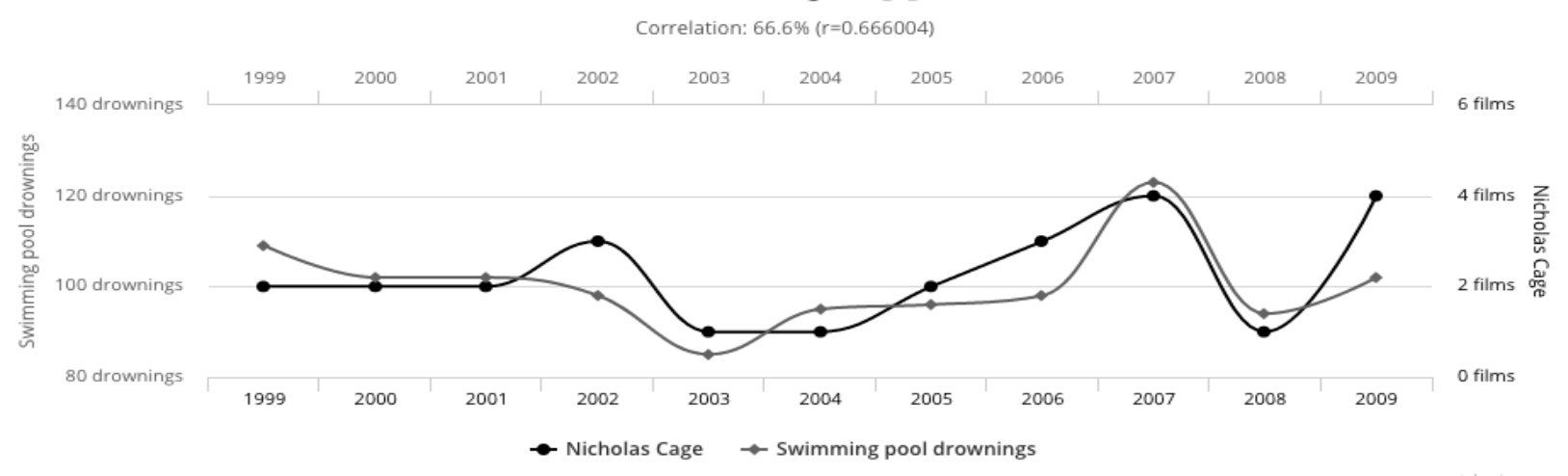

Abbildung 5. Korrelationen 
Auf der amerikanischen Website tylervigen.com finden sich völlig absurde Korrelationen, um uns vor Augen zu führen, dass zwei Kurven wunderschön parallel zueinander verlaufen können - die Annahme einer kausalen Verbindung aber gänzlicher Nonsens wäre. Oder würden Sie tatsächlich ernsthaft glauben, dass mehr Leute im Swimmingpool ertrinken, weil ein neuer Film mit Nicolas Cage erscheint? Oder dass die Selbstmordrate steigt, weil die Amerikaner mehr Geld in Weltraumforschung investieren? (Abbildung 5).

In diesen Fällen ist auf den ersten Blick evident, dass unmöglich ein kausaler Zusammenhang bestehen kann. Doch nach demselben Prinzip können auch weit weniger offensichtlich unsinnige Faktoren verquickt werden - und uns Zusammenhänge suggerieren, wo gar keine sind. Dieser unzulässigen Vermischung von Korrelation und Kausalität begegnen wir eigentlich ständig, sei es bei der Kommunikation vermeintlicher Risiken oder beim Nachweis des angeblichen Erfolgs einer Behandlungsmethode, vorzugsweise in der Alternativmedizin. Man kann beispielsweise Homöopathika einnehmen, und es geht einem anschließend besser. Das heißt aber längst nicht zwangsläufig, dass es einem besser geht, weil man Globuli geschluckt hat. Doch mit der unscharfen Trennung von Korrelation und Kausalität lässt sich gut tricksen, wenn man die Datenbasis nicht genau kennt - und es lassen sich positive (oder aber negative) Effekte vortäuschen, wo gar keine sind.

Und so könnte man in Abwandlung eines legendären Spruchs sagen: Vertrauen Sie keiner Statistik, deren Zustandekommen Sie nicht nachvollziehen können.

Acknowledgements: None.

Conflict of interest : None to declare.

Correspondence:

Alwin Schönberger

Vienna, Austria

E-mail:info@alwinschoenberger.at 